\title{
Frontières
}

\section{Mort et suicide}

\section{Jean-Dominique Leccia}

Volume 15, numéro 1, automne 2002

Délires urbains, dangers de mort

URI : https://id.erudit.org/iderudit/1073908ar

DOI : https://doi.org/10.7202/1073908ar

Aller au sommaire du numéro

Éditeur(s)

Université du Québec à Montréal

ISSN

1180-3479 (imprimé)

1916-0976 (numérique)

Découvrir la revue

\section{Citer cet article}

Leccia, J.-D. (2002). Mort et suicide. Frontières, 15(1), 60-62. https://doi.org/10.7202/1073908ar

\section{Résumé de l'article}

Dans une grande ville comme Paris, les cliniques d'urgence psychiatriques sont confrontées quotidiennement aux drames que sont les actes suicidaires. Un psychiatre nous en parle.
Ce document est protégé par la loi sur le droit d'auteur. L'utilisation des services d'Érudit (y compris la reproduction) est assujettie à sa politique d'utilisation que vous pouvez consulter en ligne.

https://apropos.erudit.org/fr/usagers/politique-dutilisation/
Cet article est diffusé et préservé par Érudit.

Érudit est un consortium interuniversitaire sans but lucratif composé de l’Université de Montréal, l’Université Laval et l'Université du Québec à Montréal. Il a pour mission la promotion et la valorisation de la recherche. https://www.erudit.org/fr/ 


\section{MORT ET SUICIDE}

\section{MAIS OÙ SONT LES CORBILLARDS D'ANTAN... ELLES SONT RÉVOLUES, ELLES ONT FAIT LEURS TEMPS, LES BELLES POMPES FUNĖBRES DE NOS VINGT ANS.}

GEORGES BRASSENS

\section{Résumé}

Dans une grande ville comme Paris, les cliniques d'urgence psychiatriques sont confrontées quotidiennement aux drames que sont les actes suicidaires. Un psychiatre nous en parle.

Mots clés : suicide - ville - urgence

\section{Abstract \\ In a large city like Paris, psychiatric emer- gency clinics are confronted daily with scenes that are acts of suicide. A psychia- trist speaks to us about this.}

Key words : suicide - city-emergency

\section{Jean-Dominique Leccia, psychiatre.}

J'ai passé les premières années de ma vie au $5^{\mathrm{e}}$ étage d'un immeuble de la banlieue parisienne. Du balcon, je regardais sur la route du cimetière passer quotidiennement les catafalques fleuris tirés par des chevaux diversement harnachés, puis les modernes corbillards plus ou moins clinquants. Dernier hommage d'une foule dense ou rachitique, ces cérémonies ont été rapidement sacrifiées au triomphe envahissant de l'automobile. Dans les métropoles, l'heure des enterrements express venait de sonner pour le commun des mortels, et les rites funéraires, hormis ceux réservés aux puissants, disparaissaient du domaine public.

La mort allait aussi déserter la sphère privée. «Jadis il n'y avait ni maison ni pièce qui n'ait vu mourir quelqu'un. [...] Aujourd'hui les bourgeois vivent dans des lieux ou personne n'est mort»(Benjamin, 2002). Walter Benjamin, qui y voit la marque d'un déclin du récit, ajoute, prophétique: «Quand ils arrivent au bout du rouleau, [ils] sont entassés par leurs héritiers dans des hôpitaux ou des sanatoriums. » Le processus est aujourd'hui finalisé, et la mort médicalisée a été largement dérobée au regard des vivants. Même si on la rencontre reléguée, dans les hôpitaux quand elle devient imminente, j'aurais moi-même pu l'oublier, les psychiatres n'ont pas de mourants en propre, et ces derniers utilisent finalement peu leurs services.

C'est pourtant en exerçant ma profession, proche de ma banlieue d'origine, que j'ai assisté à une véritable renaissance du théâtre de la mort. Je participais alors à l'implantation d'urgences psychiatriques dans divers hôpitaux parisiens. Cette pratique visait à traiter les nouvelles turbulences des villes au rythme rapide de leurs mutations. Ce type d'intervention de première ligne reproduisait celui inauguré dans les années 1950 par nos collègues de l'armée américaine en Corée. Ils devaient initialement évaluer les capacités de militaires en état de choc à retourner aux combats. De notre côté, sur les fronts urbains, nous étions sollicités pour réguler les flux, rétablir les continuités et réorienter certains sujets égarés ou les soustraire en cas d'échec au mouvement général.

Dans les années 1980, cette psychiatrie qui enregistre les écarts aux règles de la bienséance urbaine, s'installe dans les métropoles européennes. La clientèle qui défile incorpore tout ce que la ville ordinairement refoule. D'abord le sexe, celui de Pigalle dont nous sommes proches, et celui des toilettes de la Gare du Nord voisine: travestis colorés, prostitués de toute allégeance ainsi que leurs clients, dépités. Ensuite, la violence: les rixes des alcooliques ou des délinquants, les agresseurs fous, imprévisibles, et les batteurs ou battus domestiques. S'y joignent aussi les errances, les fugues d'adolescent, les voyages oniriques et la cohorte grandissante des itinérants. Enfin, la mort, le plus souvent simulée : ceux qui ont tenté de se suicider représentent $30 \%$ des consultants. Ils arrivent souvent en pleine nuit, généralement somnolents ou blessés, révoltés.

1 h 30 du matin, une jeune femme a absorbé des barbituriques : "Il y a tellement de choses, je ne veux rien dire, je veux dormir, me reposer, rentrer chez moi, j'ai déjà essayé de me suicider, je n'en parle plus. » Minuit, une jeune femme de 25 ans, 
tentative de suicide par blessure volontaire au poignet: «Ce n'est rien non, il ne se passe rien, j'ai fait une bêtise, je ne recommencerai plus, ça m'a servi de leçon. »

Ce mode de communication heurte le personnel. Il s'irrite que sa disponibilité, sa vitesse d'intervention et son efficacité soient détournés pour offrir une assistance médicale à un simulacre parfois burlesque de mort. Le corps suicidaire représente un véritable défi à l'idéal médical, il est mal toléré dans un univers où des professionnels s'acharnent à sauver les vies. Comme l'émergence de clientèles critiques aux demandes mal définies, c'est précisément la multiplication de ce type de situations conflictuelles qui a accéléré l'implantation de ces postes d'intervention psychiatrique rapide ayant pour objectif de ramasser les laissés-pour-compte, les inclassables, les riens de la médecine et de la ville.

En matière de tentatives suicidaires, nous avions la responsabilité des viatiques de sortie. Ces interventions finales pour décider des suites à donner étaient diversement appréciées : «Maintenant je vois un psychiatre, c'est le bouquet! Vous voyez bien que je ne suis pas folle. Laissez moi sortir s'il vous plaît. » Le flirt avec la mort, le plus souvent pratiqué par des jeunes femmes s'installait comme une forme de communication tragique et banalisée utilisant des techniques généralement rudimentaires, absorption plus ou moins massive de médicaments, coupures essentiellement aux poignets dans le but de s'ouvrir les veines. Des corps sans trace, parfois rattrapés au moment où ils allaient se jeter sous le métro ou sauter du train. Des corps vierges aussi où l'esquisse même du geste est absente, seul le spectre de la mort rôde. Le corps menacé ou souffrant fonctionne comme espace de représentation et ses mises en scène funèbres font le plus souvent écho à des ruptures amoureuses, des deuils, des éloignements familiaux ou territoriaux.

J'assistais ainsi, côté cour, au retour de la mort dans un environnement dont elle avait été exclue, sinon sous la forme du crime ou de l'accident ${ }^{1}$. Elle retrouvait sa puissance expressive et évocatrice, elle générait
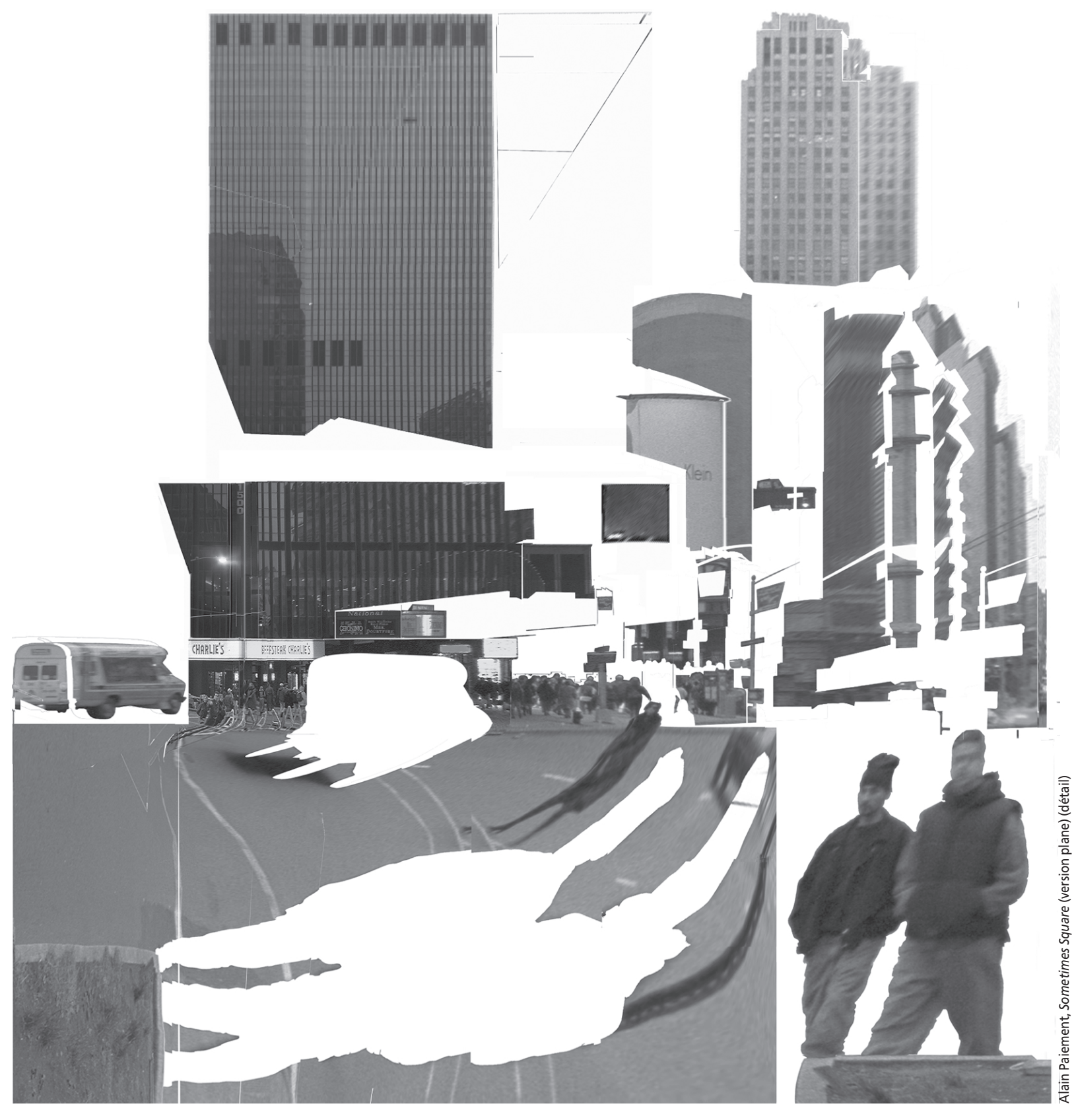
réunions familiales avec échanges de nouvelles, et conversations téléphoniques pour avoir des informations ou en donner. Plus ou moins riche et diversement partagée, cette production de récits individuels et familiaux s'autorise de la présence virtuelle de la mort, à charge pour le thérapeute d'être capable «de nommer l'innommable pour que puisse se dire l'indicible» (Ausloos, 1995). Durant ce véritable cérémonial qu'une jeune rescapée sarde encore endormie souhaiterait passer à faire la fête avec des amis sympathiques, s'exprime parfois pour la première fois la souffrance de suicidaires repentis ou entêtés et celle de leur entourage épuisé. La jeune fille à moitié endormie a pris des comprimés. Son ami, garçon de café, ne la comprend plus : «Elle revient de chez son père, dit-il, et moi j'ai vu ma fille cet après-midi. Elle est jalouse et capricieuse. Tous les soirs après mon travail, elle veut que l'on sorte et que l'on fasse l'amour. Moi, j'ai dix ans de plus qu'elle, alors je craque. » Le prince charmant abdique et rentre seul à la maison tandis qu'elle, muette comme la tombe, repart clandestinement de bonne heure pour le rejoindre.

C'est un lieu où les récits foisonnent et ces scénarisations symptomatiques réintroduisent dans l'univers urbain ce dont il avait cru s'affranchir, le spectacle de la mort en direct $^{2}$. Cette économie de la mort qui la rend invisible avec, comme dernier interlocuteur, la médecine croise une expansion mondiale du nombre des suicides. Différente, la tentative suicidaire est plus localisée, civilisationnelle, le modèle est urbain et occidental. En témoigne son adoption transgressive par des jeunes femmes de l'immigration. La manière dont elles s'emparent de cette modalité de communication passant par une théâtralisation de la mort opère comme un acte d'allégeance territoriale. L'acte suicidaire crée un véritable choc culturel qui mobilise d'abord les femmes de la famille, supportantes mais soucieuses de préserver le silence sur un tel acte vécu comme une trahison. Cette modalité d'expression, avec ses marqueurs culturels propres tend à se répandre aujourd'hui dans toutes les métropoles de la planète au rythme de la mondialisation et de l'occidentalisation des espaces, tant réels que médiatiques.

Au moment où le corps revient en première ligne pour exprimer ce que la ville dissimule, l'image médiatique prend le relais et consacre le spectacle de la mort. Ce sont ses mises en scène qui aujourd'hui écrivent les légendes et renouvellent les récits, depuis la répétition de funérailles de stars internationales jusqu'au deuil brutal de figures emblématiques comme celle de la Princesse Diana, morte en vitesse, sur les berges de la Seine. Au-delà des espaces médiatiques où l'image elle-même peut dangereusement se faire chair ${ }^{3}$ naissent, aujourd'hui, des espaces virtuels prenant en charge le fardeau de la mort. Les sites funéraires se multiplient sur le Web ce qui, avec la crémation des corps, achèvent la « ringardisation » du cimetière traditionnel.
Ce mouvement d'abstraction de la mort qui l'affranchit des contraintes physiques et géographiques suscite des craintes plus diffuses. Elles s'expriment à l'urgence sous forme d'angoisse, de panique qui ne s'adressent plus à une mort individualisée mais civilisationnelle. C'est la survie de l'espace urbain lui-même qui est en question, engendrant dans ces formes extrêmes de véritables psychoses territoriales.

\section{Bibliographie}

AUSLOOS, G. (1995). La compétence des familles, Érès.

BENJAMIN, Walter (2002). CEuvres III, Folio.

BENJAMIN, Walter (1988). Sens unique, $1018 \mathrm{p}$.

\section{Notes}

1. «S'ils surgissaient, ils avaient déjà disparu quand j'arrivais sur les lieux, comme des dieux qui ne disposent que de quelques instants pour les mortels »(Benjamin, 1988, p. 130).

2. On garde en mémoire les rares images des défenestrés ou des blessés du World Trade Center, en opposition avec la débauche de corps morts et mutilés, exhibés aux heures de grande écoute dès qu'il s'agit d'univers étrangers. Le refoulement s'associerait-il à la projection ou, en termes plus prosaïques, à l'exportation?

3. Au moment où la réalité rejoint les fictions, l'image perd toute distance, comme on a pu le constater à New York, le 11 septembre 2001. 